\title{
Effective-mass theory for InAs/GaAs strained coupled quantum dots
}

\author{
Shu-Shen Li,* Jian-Bai Xia, Z. L. Yuan,* and Z. Y. Xu \\ China Center of Advanced Science and Technology (World Laboratory), P.O. Box 8730, Beijing 100080, China \\ and National Laboratory for Superlattices and Microstructures, Institute of Semiconductors, Chinese Academy of Sciences, \\ P.O. Box 912, Beijing 100083, China
}

\author{
Weikun Ge, Xiang Rong Wang, Y. Wang, J. Wang, and L. L. Chang \\ Department of Physics, Hong Kong University of Science \& Technology, Clear Water Bay, Kowloon, Hong Kong
}

(Received 23 January 1996; revised manuscript received 6 May 1996)

\begin{abstract}
In the framework of effective-mass envelope-function theory, the optical transitions of InAs/GaAs strained coupled quantum dots grown on GaAs (100) oriented substrates are studied. At the $\Gamma$ point, the electron and hole energy levels, the distribution of electron and hole wave functions along the growth and parallel directions, the optical transition-matrix elements, the exciton states, and absorption spectra are calculated. In calculations, the effects due to the different effective masses of electrons and holes in different materials are included. Our theoretical results are in good agreement with the available experimental data. [S0163-1829(96)09339-3]
\end{abstract}

\section{INTRODUCTION}

Growth of highly strained InAs/GaAs quantum-well heterostructures has attracted much attention due to their unique electronic and optical properties as well as potential applications in electronic and optoelectronic devices. ${ }^{1-4}$ More recently there has arisen an interest towards structures with further reduced dimensionality: quantum wires and dots. InAs quantum wires and dots have been grown selectively on $\mathrm{SiO}_{2}$-patterned $\mathrm{GaAs}$ substrates and on terraced $\mathrm{GaAs}$ surfaces with InAs monolayer (ML) coverage. ${ }^{5,6}$ The growth of highly strained semiconductor layer (InAs, $\operatorname{In}_{x} \mathrm{Ga}_{1-x} \mathrm{As}$ ) onto a substrate (GaAs, InP) could lead to the spontaneous formation of semiconductor nanometer-scale clusters. ${ }^{7-13}$ Very sharp emission lines from InAs/GaAs heterostructures prepared from this method have been observed recently, ${ }^{14-16}$ directly representing the $\delta$-function-like density of states in the zero-dimensional system.

P. D. Wang et al. ${ }^{17}$ studied the optical properties of InAs/ GaAs heterostructures with InAs average layer thickness ranging from $1 \AA$ (one-third of a ML) to 4 ML grown on (100) and (311) surfaces. Extremely high optical quality was revealed for the structures with ultrasmall InAs coverage. Optical anisotropy found in photoluminescence (PL) as well as in PL excitation spectra indicates a highly anisotropic growth mode for InAs molecules on the GaAs (100) surface. The 1- $\AA$ sample had the narrowest $(\cong 0.8 \mathrm{meV})$ linewidth, which was consistent with the formation of $1 \mathrm{ML}$ islands with lateral dimension comparable to exciton Bohr radius and implied a uniform distribution of InAs molecules on the GaAs surface. Another remarkable feature of the $1 \AA$ sample was the extremely high efficiency of radiative recombination, especially at high temperatures. This result clearly indicates a dramatic reduction of nonradiative recombination channels.

Xie et $_{\text {al. }}{ }^{18}$ demonstrated a vertically self-organized growth of InAs quantum box islands. A phenomenological model was also proposed. The model was shown to consis- tently account for the observations, thus providing the first clear evidence that the island-induced evolving strain fields provide the driving force for self-assembly or selforganization in island systems. The strong vertically selforganized islands open a new front for studying the properties of nanostructures, such as the coupled InAs quantum boxes.

For the theoretical approach, Burt ${ }^{19}$ developed an envelope-function theory that is suitable for every kind of microstructure. He derived an effective-mass equation under only one condition that the envelope function is slowly varying. By focusing on the equation satisfied by slowly varying envelope functions, it becomes clear why the effective-mass approximation works so well for quantum wells with only 1 ML or so wide, in which the electronic states are weakly bound and thus necessarily extended. Foreman ${ }^{20,21}$ derived the conduction-band effective-mass differential equation and the valence-band effective-mass Hamiltonian of a semiconductor quantum well or superlattice. It is shown that the correct form of the valence-band effective-mass Hamiltonian gives reasonable physical results, while the commonly used "symmetrized" form can produce nonphysical solutions for the heavy-hole $(\mathrm{HH})$ subbands in which the quantum-well effective mass is very sensitive to the difference in Luttinger parameters between the well and the barrier.

In this paper, using the effective-mass envelope-function theory, we will study the electron and hole energy levels at the $\Gamma$ point, the distribution of electron and hole wave functions along the growth and parallel directions, the optical transition matrix elements, the excitonic states, and the optical-absorption spectra of coupled InAs/GaAs strained quantum dots grown on $\mathrm{GaAs}(100)$ oriented substrates by the plane-wave expansion method. ${ }^{22,23}$ In our calculations, the effects due to the different effective masses of electrons and holes in different materials, and the coupling between $\mathrm{HH}$ and light hole (LH) are included. Presently, to our knowledge, there is no suitable theory for this problem. 

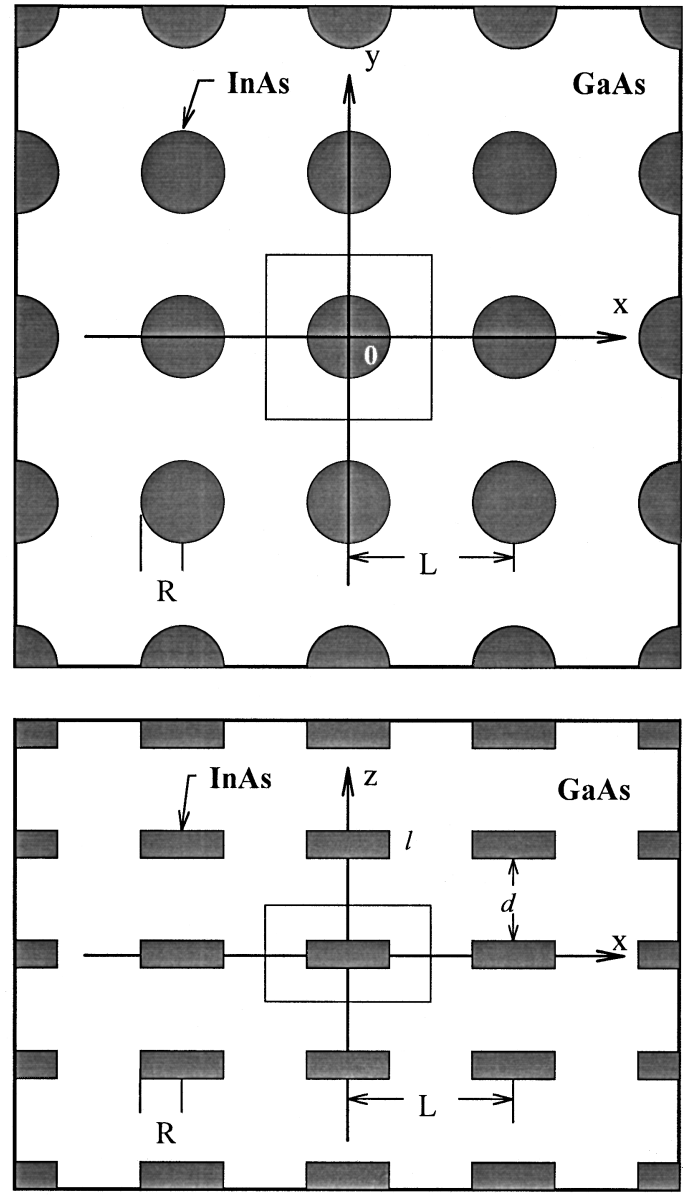

FIG. 1. The structures of coupled InAs monolayer quantum dots: (a) along the parallel direction and (b) along the growth direction.

\section{THEORETICAL MODEL}

Studying the case of the InAs/GaAs coupled quantum dots grown on (100) GaAs substrates, we assume that the strain only arises in the InAs dots. In the following we choose the growth direction (100) as the $Z$ direction of our coordinate system. The InAs dots are periodically arranged boxes. ${ }^{18}$ In the $Z$ direction, the width of the InAs dot is $l$, and the distance between two adjacent dots is $d$, the period is then $l+d$. In the parallel direction, the radius of InAs dots is $R$, the distance between two nearby dots is $L-2 R$, and $L$ is the period. We choose the boxed region as the unit cell in Fig. 1.

According to Burt and Foreman's effective-mass theory and taking into account the differences of the effective masses between InAs and GaAs materials, ${ }^{19,20}$ the electron Hamiltonian can be written as the equation below (neglecting the second- and higher-order terms in the approximation),

$$
H_{e}=\mathbf{P} \frac{1}{2 m_{e}^{*}(x, y, z)} \mathbf{P}+V_{e}(x, y, z) .
$$

In the unit cell in Fig. 1,

$$
\begin{gathered}
V_{e}(x, y, z)=\left\{\begin{array}{ll}
0, & x^{2}+y^{2} \leqslant R^{2} \\
V_{e 0}, & \text { others, }
\end{array} \text { and }|z| \leqslant l\right. \\
m_{e}^{*}(x, y, z)=\left\{\begin{array}{ll}
m_{1}^{*}, & x^{2}+y^{2} \leqslant R^{2} \\
m_{2}^{*}, & \text { others, }
\end{array} \text { and }|z| \leqslant l\right.
\end{gathered}
$$

$m_{1}^{*}$ and $m_{2}^{*}$ are the electron effective masses in InAs and GaAs materials, respectively. The electron Schrödinger equation is

$$
H_{e} \Psi_{e}=E_{e} \Psi_{e} .
$$

Assuming that the electron wave functions have the following forms:

$$
\Psi_{e}\left(\mathbf{r}_{e}\right)=\frac{1}{L \sqrt{l+d}} n_{x}, n_{y}, n_{z} e_{n_{x}, n_{y}, n_{z}} e^{i\left(k_{n z} x+k_{n y} y+k_{n z} z\right)},
$$

with $\quad k_{n x}=k_{x}+n_{x} K_{x}, \quad k_{n y}=k_{y}+n_{y} K_{y}, \quad k_{n z}=k_{z}+n_{z} K_{z}$, $K=K_{x}=K_{y}=2 \pi / L, \quad K_{z}=2 \pi /(l+d), \quad \mathbf{r}=\left(\mathbf{r}_{I I}, z\right)$, $\mathbf{r}_{I I}=(x, y)$, and $n_{x}, n_{y}, n_{z}=0, \pm 1, \pm 2, \ldots$, the matrix elements of Hamiltonian (1) for Eq. (4) can be written as

$$
\begin{aligned}
& \left(\frac{\hbar^{2}}{2 m_{2}^{*}} \delta_{n_{x} n_{x}^{\prime}} \delta_{n_{y} n_{y}^{\prime}} \delta_{n_{z} n_{z}^{\prime}}+\frac{\hbar^{2}}{2 m_{12}^{*}} S_{i} S_{j}\right) \\
& \quad \times\left(k_{n x} k_{n x}^{\prime}+k_{n y} k_{n y}^{\prime}+k_{n z} k_{n z}^{\prime}\right) \\
& \quad+\left(\delta_{n_{x_{x} n_{x}^{\prime}}} \delta_{n_{y} n_{y}^{\prime}} \delta_{n_{z} n_{z}^{\prime}}-S_{i} S_{j}\right) V_{e 0},
\end{aligned}
$$

where

$$
\begin{aligned}
& k_{n x}^{\prime}=k_{x}+n_{x}^{\prime} K_{x}, \quad k_{n y}^{\prime}=k_{y}+n_{y}^{\prime} K_{y}, \quad k_{n z}^{\prime}=k_{z}+n_{z}^{\prime} K_{z}, \\
& \frac{\hbar^{2}}{2 m_{12}^{*}}=\frac{\hbar^{2}}{2 m_{1}^{*}}-\frac{\hbar^{2}}{2 m_{2}^{*}}, \\
& S_{i}=\left\{\begin{array}{l}
\frac{l}{l+d}, \quad n_{z}=n_{z}^{\prime} \\
\frac{\sin \left[\pi\left(n_{z}-n_{z}^{\prime}\right) \frac{l}{l+d}\right]}{\pi\left(n_{z}-n_{z}^{\prime}\right)}, \quad n_{z} \neq n_{z}^{\prime},
\end{array}\right. \\
& S_{j}=\left\{\begin{array}{l}
\frac{\pi R^{2}}{L^{2}}, \quad n_{x}=n_{x}^{\prime} \text { and } n_{y}=n_{y}^{\prime} \\
\frac{R}{\lambda L} J_{1}(\lambda K R), \quad n_{x} \neq n_{x}^{\prime} \text { or } n_{y} \neq n_{y}^{\prime},
\end{array}\right.
\end{aligned}
$$

and

$$
\lambda=\sqrt{\left(n_{x}-n_{x}^{\prime}\right)^{2}+\left(n_{y}-n_{y}^{\prime}\right)^{2}} .
$$

$J_{1}$ is the first-order Bessel function, $J_{1}(x)$ $=(x / 2 \pi) \int_{0}^{\pi} \cos (x \cos \theta) \sin ^{2} \theta d \theta$. Therefore, we can calculate the electron subband structures from Eq. (5).

For the valence subbands, including the effects of strain, the hole Hamiltonian can be written as (assuming the hole energy as positive)

$$
H_{h}=H_{0}+H_{\varepsilon}+V_{h}(x, y, z) .
$$


$V_{h}(x, y, z)$ is the hole potential of coupled quantum dots. In the unit cell in Fig. 1,

$$
V_{h}(x, y, z)=\left\{\begin{array}{l}
0, \quad x^{2}+y^{2} \leqslant R^{2} \text { and }|z| \leqslant l \\
V_{h 0}, \quad \text { others, }
\end{array}\right.
$$

$H_{0}$ is the Foreman effective-mass Hamiltonian for the hole state (excluding spin-orbit splitting) ${ }^{21}$

$$
H_{0}=\frac{1}{2 m_{0}}\left[\begin{array}{cccc}
P_{+} & R & -Q_{-} & 0 \\
R^{+} & P_{-} & C^{+} & -Q_{+}^{+} \\
-Q_{-}^{+} & C & P_{-} & -R \\
0 & -Q_{+} & -R^{+} & P_{+}
\end{array}\right] \text {, }
$$

with

$$
\begin{aligned}
& P_{ \pm}=\hat{p}_{x}\left(\gamma_{1} \pm \gamma_{2}\right) \hat{p}_{x}+\hat{p}_{y}\left(\gamma_{1} \pm \gamma_{2}\right) \hat{p}_{y}+\hat{p}_{z}\left(\gamma_{1} \mp 2 \gamma_{2}\right) \hat{p}_{z}, \\
& R=\sqrt{3}\left[\left(\hat{p}_{x}+i \hat{p}_{y}\right) \mu\left(\hat{p}_{x}+i \hat{p}_{y}\right)-\left(\hat{p}_{x}-i \hat{p}_{y}\right) \bar{\gamma}\left(\hat{p}_{x}-i \hat{p}_{y}\right)\right],
\end{aligned}
$$

$$
\begin{gathered}
Q_{ \pm}=2 \sqrt{3}\left[\left(\hat{p}_{x} \pm i \hat{p}_{y}\right)(\sigma-\delta) \hat{p}_{z}+\hat{p}_{z} \pi\left(\hat{p}_{x} \pm i \hat{p}_{y}\right)\right], \\
C=2 \hat{P}_{z}(\sigma-\delta-\pi)\left(\hat{p}_{x}-i \hat{p}_{y}\right)-2\left(\hat{p}_{x}-i \hat{p}_{y}\right)(\sigma-\delta-\pi) \hat{P}_{z}, \\
\sigma-\delta=\frac{1}{6}\left(-1-\gamma_{1}+2 \gamma_{2}+6 \gamma_{3}\right), \quad \pi=\frac{1}{6}\left(1+\gamma_{1}-2 \gamma_{2}\right), \\
\bar{\gamma}=\frac{1}{2}\left(\gamma_{2}+\gamma_{3}\right), \text { and } \mu=-\frac{1}{2}\left(\gamma_{2}-\gamma_{3}\right) .
\end{gathered}
$$

$\gamma_{1}, \gamma_{2}, \gamma_{3}$ are the Luttinger effective-mass parameters which are functions of $x, y, z$. In the unit cell in Fig. 1,

$$
\begin{aligned}
& \gamma_{1}, \gamma_{2}, \gamma_{3}(x, y, z) \\
& =\left\{\begin{array}{c}
\gamma_{11}, \gamma_{12}, \gamma_{13}, \quad x^{2}+y^{2} \leqslant R^{2} \quad \text { and }|z| \leqslant l, \\
\gamma_{21}, \gamma_{22}, \gamma_{23}, \quad \text { others, }
\end{array}\right.
\end{aligned}
$$

where $\gamma_{11}, \gamma_{12}, \gamma_{13}$ and $\gamma_{21}, \gamma_{22}, \gamma_{23}$ are the Luttinger effective-mass parameters of InAs and GaAs, respectively. $m_{0}$ is the free-electron effective mass. $H_{\varepsilon}$ is the strained energies of the hole $\mathrm{e}^{24,25}$

$$
\begin{aligned}
H_{\varepsilon}= & -D_{d}\left(\varepsilon_{x x}+\varepsilon_{y y}+\varepsilon_{z z}\right)-\frac{2}{3} D_{u}\left[\left(J_{x}^{2}-\frac{1}{3} J^{2}\right) \varepsilon_{x x}+\left(J_{y}^{2}-\frac{1}{3} J^{2}\right) \varepsilon_{y y}+\left(J_{z}^{2}-\frac{1}{3} J^{2}\right) \varepsilon_{z z}\right] \\
& -\frac{2}{3} D_{u}^{\prime}\left[2\left\{J_{x}, J_{y}\right\} \varepsilon_{x x}+2\left\{J_{y}, J_{z}\right\} \varepsilon_{y z}+2\left\{J_{z}, J_{x}\right\} \varepsilon_{z x}\right],
\end{aligned}
$$

where $D_{d}, D_{u}, D_{u}^{\prime}$ are the deformation potentials, $\varepsilon_{x x}, \varepsilon_{y y}, \ldots$ are the strain tensor components. The substrate of the InAs dots is GaAs, we assume

$$
\varepsilon_{x x}=\varepsilon_{y y}=\frac{a_{0}-a}{a} \text { and } \varepsilon_{i j}=0 \quad(i \neq j),
$$

in InAs where $a$ and $a_{0}$ are the lattice parameters of bulk InAs and GaAs, respectively. The energy density of strain is $U=\frac{1}{2} C_{11}\left(2 \varepsilon_{x x}^{2}+\varepsilon_{z z}^{2}\right)+C_{12}\left(2 \varepsilon_{x x} \varepsilon_{z z}+\varepsilon_{x x}^{2}\right) . U$ should have minimum in the condition (11), so

$$
\varepsilon_{z z}=-2 \frac{C_{12}}{C_{11}} \varepsilon_{x x}
$$

$C_{12}$ and $C_{11}$ are the elastic moduli of InAs. Using the representation of $J_{x}, J_{y}$, and $J_{z}$ in Eq. (39) of Ref. 26, we obtain the hole effective-mass Hamiltonian,

$$
\begin{aligned}
H_{h}= & \frac{1}{2 m_{0}}\left[\begin{array}{cccc}
P_{+}-D & R & -Q_{-} & 0 \\
R^{+} & P_{-}+D & C^{+} & -Q_{+}^{+} \\
-Q_{-}^{+} & C & P_{-}+D & -R \\
0 & -Q_{+} & -R^{+} & P_{+}-D
\end{array}\right]+D^{\prime} \\
& +V_{h}\left(z_{h}\right),
\end{aligned}
$$

where

$$
D=\left\{\begin{array}{l}
D_{0}, \quad x^{2}+y^{2} \leqslant R^{2} \text { and }|z| \leqslant l, \\
0, \quad \text { others, }
\end{array}\right.
$$

$$
\begin{gathered}
D_{0}=-\frac{2}{3} D_{u}\left(1+\frac{2 c_{12}}{c_{11}}\right) \varepsilon_{x x}, \\
D^{\prime}=-2 D_{d}\left(1-\frac{c_{12}}{c_{11}}\right) \varepsilon_{x x} .
\end{gathered}
$$

Assuming the hole wave function has the following form:

$$
\Psi_{h}\left(\mathbf{r}_{h}\right)=\frac{1}{L \sqrt{l+d}} n_{x}, n_{y}, n_{z}\left(\begin{array}{c}
a_{n_{x}, n_{y}, n_{z}} \\
b_{n_{x}, n_{y}, n_{z}} \\
c_{n_{x}, n_{y}, n_{z}} \\
d_{n_{x}, n_{y}, n_{z}}
\end{array}\right) e^{i\left(k_{n x} x+k_{n y} y+k_{n z} z\right)},
$$

the matrix elements of Hamiltonian (13) between the wave function (15) are then

$$
\begin{aligned}
\left(P_{ \pm} \mp D\right)_{n n^{\prime}}= & \left(\gamma_{ \pm}^{1} \delta_{n_{x} n_{x}^{\prime}} \delta_{n_{y} n_{y}^{\prime}} \delta_{n_{z} n_{z}^{\prime}}+S_{i} S_{j} \gamma_{ \pm}^{2}\right) \\
& \times\left(k_{n x} k_{n x}^{\prime}+k_{n y} k_{n y}^{\prime}\right) \\
& +\left(\gamma_{ \pm}^{3} \delta_{n_{x} n_{x}^{\prime}} \delta_{n_{y} n_{y}^{\prime}} \delta_{n_{z} n_{z}^{\prime}}+S_{i} S_{j} \gamma_{ \pm}^{4}\right) \\
& \times k_{n z} k_{n z}^{\prime} \mp D_{0} S_{i} S_{j}
\end{aligned}
$$




$$
\begin{aligned}
&\left(Q_{ \pm}\right)_{n n^{\prime}}= 2 \sqrt{3}\left\{\left[\delta_{n_{x} n_{x}^{\prime}} \delta_{n_{y} n_{y}^{\prime}} \delta_{n_{z} n_{z}^{\prime}}\left(\sigma_{2}-\delta_{2}\right)\right.\right. \\
&\left.+\left(\sigma_{1}-\delta_{1}-\sigma_{2}+\delta_{2}\right) S_{i} S_{j}\right]\left(k_{n x}^{\prime} \pm i k_{n y}^{\prime}\right) k_{n z} \\
&+\left[\delta_{n_{x} n_{x}^{\prime}} \delta_{n_{y} n_{y}^{\prime}} \delta_{n_{z} n_{z}^{\prime}} \pi_{2}+\left(\pi_{1}-\pi_{2}\right) S_{i} S_{j}\right] \\
&\left.\times\left(k_{n x} \pm i k_{n y}\right) k_{n z}^{\prime}\right\}, \\
& R_{n n^{\prime}}= \sqrt{3}\left\{\left[\delta_{n_{x} n_{x}^{\prime}} \delta_{n_{y} n_{y}^{\prime}} \delta_{n_{z} n_{z}^{\prime}} \mu_{2}+\left(\mu_{1}-\mu_{2}\right) S_{i} S_{j}\right]\left(k_{n x}+i k_{n y}\right)\right. \\
& \times\left(k_{n x}^{\prime}+i k_{n y}^{\prime}\right)-\left[\delta_{n_{x} n_{x}^{\prime}} \delta_{n_{y} n_{y}^{\prime}} \delta_{n_{z} n_{z}} \bar{\gamma}_{2}+\left(\bar{\gamma}_{1}-\bar{\gamma}_{2}\right) S_{i} S_{j}\right] \\
&\left.\times\left(k_{n x}-i k_{n y}\right)\left(k_{n x}^{\prime}-i k_{n y}^{\prime}\right)\right\}, \\
& C_{n n^{\prime}}=2\left[\left(k_{n x}-i k_{n y}\right) k_{n z}^{\prime}-\left(k_{n x}^{\prime}-i k_{n y}^{\prime}\right) k_{n z}\right] \\
& \times\left(\sigma_{1}-\delta_{1}-\pi_{1}-\sigma_{2}+\delta_{2}+\pi_{2}\right) S_{i} S_{j}, \quad(16 \mathrm{~d}) \\
&\left(D^{\prime}+V_{h}\right)_{n n^{\prime}}=D^{\prime} S_{i} S_{j}+\left(\delta_{n_{x} n_{x}^{\prime}} \delta_{n_{y} n_{y}^{\prime}} \delta_{n_{z} n_{z}^{\prime}}-S_{i} S_{j}\right) V_{h 0},
\end{aligned}
$$

with $\quad \gamma_{ \pm}^{1}=\gamma_{21} \pm \gamma_{22}, \quad \gamma_{ \pm}^{2}=\left(\gamma_{11} \pm \gamma_{12}\right)-\gamma_{ \pm}^{1}, \quad \gamma_{ \pm}^{3}=\gamma_{21}$ $\mp 2 \gamma_{22}, \quad \gamma_{ \pm}^{4}=\left(\gamma_{11} \mp 2 \gamma_{12}\right)-\gamma_{ \pm}^{3}, \quad \sigma_{n}-\delta_{n}=\frac{1}{6}\left(-1-\gamma_{n 1}\right.$ $\left.+2 \gamma_{n 2}+6 \gamma_{n 3}\right), \pi_{n}=\frac{1}{6}\left(1+\gamma_{n 1}-2 \gamma_{n 2}\right), \bar{\gamma}_{n}=\frac{1}{2}\left(\gamma_{n 2}+\gamma_{n 3}\right)$, $\mu_{n}=-1 / 2\left(\gamma_{n 2}-\gamma_{n 3}\right), \quad \sigma_{n}-\delta_{n}-\pi_{n}=\frac{1}{3}\left(-1-\gamma_{n 1}+2 \gamma_{n 2}\right.$ $\left.+3 \gamma_{n 3}\right)$, and $n=1$ or 2 . The hole subband structures can be calculated from Eq. (16).

The treatment of strain effects in Eqs. (11) and (12) is a good approximation for large $R$, but in the limit of small $R$, the strain will approach the case of $e_{x x}=e_{y y}=e_{z z}=\left(a_{0}-a\right) / a$. So, our simplified treatment of strain will break down for small $R$. Due to the matrix elements of strain energy include the factor $S_{j}$ [Eqs. (16a) and (16e)] and $S_{j}$ will approach zero when $R$ approaches zero; the transition energies calculated using Eq. (12) is close to those calculated using $e_{z z}=e_{x x}=e_{y y}$ for the small $R$. The $R=0$ transition energies is equal to the transition energies of bulk GaAs.

The exciton Hamiltonian can be written as

$$
H=H_{e}+H_{h}-\frac{e^{2}}{\varepsilon|\mathbf{r}|} .
$$

Assuming that the exciton wave function is of the following form:

$$
\Psi_{e x}=\Psi_{e} \Psi_{h} G(\rho, z, \theta)=|i, j\rangle,
$$

where

$$
\begin{gathered}
G(\rho, z, \theta)=\sum_{i j} A_{i j}\left(\frac{2 \alpha_{i}}{\pi}\right)^{1 / 2}\left(\frac{2 \beta_{j}}{\pi}\right)^{1 / 4} \exp \left(-\alpha_{i} \rho^{2}-\beta_{j} z^{2}\right), \\
\rho^{2}=\left(x_{e}-x_{h}\right)^{2}+\left(y_{e}-y_{h}\right)^{2} \text {, and } \\
z^{2}=\left(z_{e}-z_{h}\right)^{2} .
\end{gathered}
$$

The exciton energies can then be determined by

$$
\operatorname{det}\left(H_{i^{\prime} j^{\prime}, i j}-E S_{i^{\prime} j^{\prime}, i j}\right)=0,
$$

with
TABLE I. The effective masses and lattice parameters.

\begin{tabular}{lccccc}
\hline \hline & $m_{e}^{*}\left(m_{0}\right)$ & $\gamma_{1}$ & $\gamma_{2}$ & $\gamma_{3}$ & $a_{0}(\AA)$ \\
\hline GaAs & 0.067 & 6.85 & 2.1 & 2.9 & 5.653 \\
InAs & 0.023 & 19.67 & 8.37 & 9.29 & 6.0583 \\
\hline \hline
\end{tabular}

$$
H_{i^{\prime} j^{\prime}, i j}=\left\langle i^{\prime} j^{\prime}\left|H_{e}+H_{h}-\frac{e^{2}}{\varepsilon|\mathbf{r}|}\right| i j\right\rangle,
$$

and $S_{i^{\prime} j^{\prime}, i j}=\left\langle i^{\prime} j^{\prime} \mid i j\right\rangle$. The exciton binding energies are therefore given by

$$
E_{b}=E_{e}+E_{h}-E .
$$

The absorption coefficients for interband transitions can be written as ${ }^{27,28}$

$$
\alpha(\hbar \omega)=\frac{C}{\omega} \sum_{i}\left|\left\langle\Psi_{e x}|\hat{\varepsilon} \cdot \mathbf{P}| G\right\rangle\right|^{2} \delta(E-\hbar \omega),
$$

where $C$ is a constant and $G$ denotes the ground state of the solid. Assuming that the momentum matrix elements between the conduction- and valence-band Bloch states are independent of $\mathbf{k},{ }^{28}$ we obtain

$$
\left\langle\Psi_{e x}|\hat{\varepsilon} \cdot \mathbf{P}| G\right\rangle=Q_{m m^{\prime}} \sum_{\mathbf{k}} G(\mathbf{k}),
$$

where $Q_{m m^{\prime}}$ is the squared optical transition matrix elements.

In order to obtain a smooth absorption spectrum, we replace the $\delta$ function in Eq. (21) by a Lorentzian function with a half-width $\Gamma$, viz.,

$$
\delta(E-\hbar \omega) \approx \frac{\Gamma}{\pi\left[(E-\hbar \omega)^{2}+\Gamma^{2}\right]} .
$$

The magnitude of $\Gamma$ is roughly equal to the energy spacing of the eigenstates.

\section{RESULTS AND DISCUSSION}

The number of plane waves that were used in our calculations are $n_{x}, n_{y}, n_{z}=0, \pm 1, \pm 2, \pm 3$. Taking more plane waves, the calculated results will be slightly improved.

When $l=30 \AA, d=50 \AA, R=50 \AA$, and $L=200 \AA$, we take the effective masses and lattice parameters in Table I. ${ }^{29}$ The conduction-band offset is assumed to be $70 \%$ of the band-gap difference. ${ }^{17}$ The band offsets are given in Table II. For InAs materials, we take the deformation potential parameters: $D_{d}=-a=6.0 \mathrm{eV}, 2 D_{u} / 3=-b=1.8 \mathrm{eV}$, and the elastic moduli (in $10^{11} \mathrm{dyn} \mathrm{cm}^{-2}$ ): $c_{11}=8.329, c_{12}=4.526 .{ }^{29} \mathrm{We}$ have calculated the electron and hole subbands along the

TABLE II. The band offset parameters in strained and unstrained values (in units of $\mathrm{eV}$ ).

\begin{tabular}{lcccc}
\hline \hline & $E_{G(\mathrm{GaAs})}$ & $E_{G(\mathrm{InAs})}$ & $V_{e 0}$ & $V_{h 0}$ \\
\hline Unstrained & 1.518 & 0.418 & 0.77 & 0.33 \\
Strained & 1.518 & 0.78456 & 0.51341 & 0.22003 \\
\hline
\end{tabular}


TABLE III. The energy levels $(\mathrm{meV})$ at the $\Gamma$ point $(k=0)$.

\begin{tabular}{lcccc}
\hline \hline & $m^{*}$ & $m^{*}=m_{1}^{*}$ & $m_{1}^{*}=m_{2}^{*}$ & $m^{*}=m_{2}^{*}$ \\
\hline First & Conductive & 417.1 & 365.8 & 281.6 \\
HH1 & mixed & 100.0 & 79.9 & 80.4 \\
& not mixed & 155.7 & 129.5 & 100.6 \\
& & & & \\
LH1 & mixed & 103.1 & 84.4 & 91.7 \\
& not mixed & 172.4 & 150.3 & 131.3 \\
\hline \hline
\end{tabular}

growth direction (100) and the parallel direction of GaAs/ InAs quantum dots. The results indicate narrow bandwidths and sharp peak density of states in coupled quantum dots. The width of the first band is only about $1 \mathrm{meV}$ for both electron and hole. The width of bands will become larger when the dots are closer to each other or the dots are wider in size. The mixing between the $\mathrm{LH}$ and $\mathrm{HH}$ is determined by $R, Q$, and $C$ in Eq. (8). The mixing still exists even when $k=0$ (at the $\Gamma$ point) in coupled quantum dots, while it does not exist in superlattices. The energy levels at the $\Gamma$ point $(k=0)$ are shown in Table III. From this table, we can see that the energy levels are strongly affected by the material parameters and the mixing effect between $\mathrm{LH}$ and $\mathrm{HH}$ in coupled quantum dots. Using the envelope-function approach, one can indeed show that the excitonic wave functions are mostly confined in InAs dots. For simplicity, we assume here an effective mass, close to that of the InAs, for the whole InAs-GaAs coupled quantum dots system, and perform the same effective-mass calculation. When the volume of the InAs dots is not very small, this is a good ap-
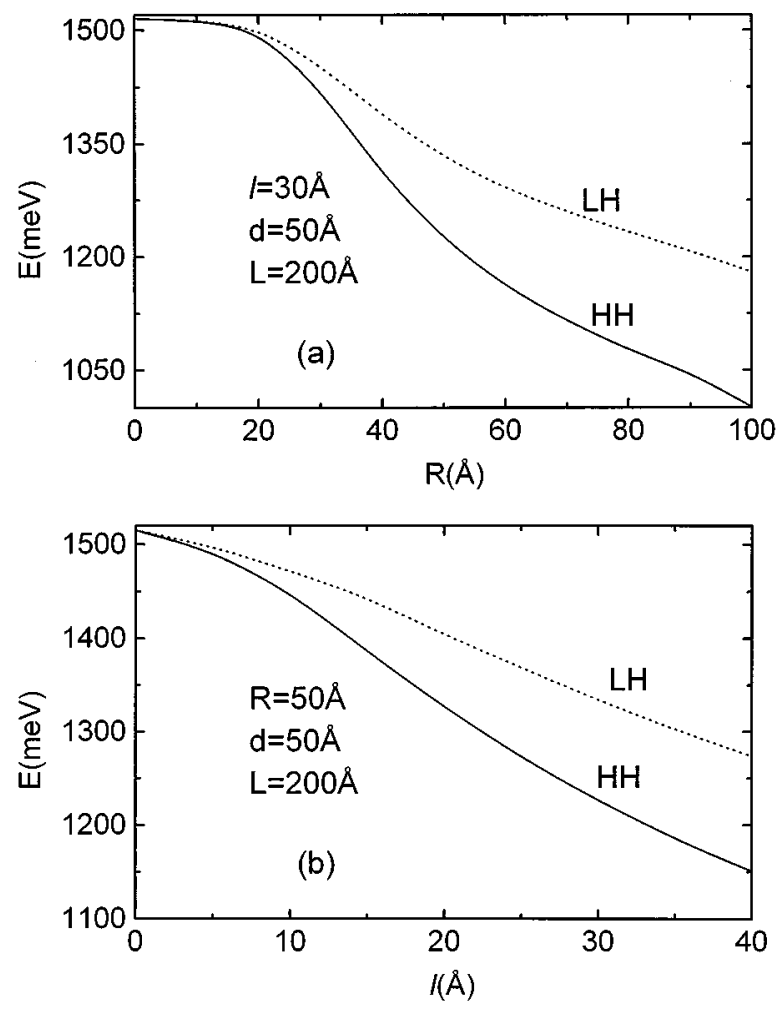

FIG. 2. The heavy- and light-hole energy transitions as functions of the radius $R$ (a) and width $l$ (b).
TABLE IV. The squared optical transition matrix elements from the first electronic energy level to the first heavy-hole and light-hole energy levels.

\begin{tabular}{lccc}
\hline \hline & Model & $Q_{z}$ & $Q_{\|}$ \\
\hline HH1 & quantum dots & 0 & 18.156 \\
& superlattices & 0 & 17.079 \\
& & & \\
LH1 & quantum dots & 24.499 & 6.125 \\
& superlattices & 24.853 & 6.214 \\
\hline \hline
\end{tabular}

proximation. Including the exciton effects, Fig. 2 gives the $\mathrm{HH}$ and LH energy transitions as functions of the radius $R$ (a) and width $L$ (b). The energy transition will decrease as the size of InAs dots increase.

For the monolayer coupled quantum dots, $l=2.83 \AA$ A . Setting $k=0$, and taking above structure parameters, as well as the optical transition matrix constant $2 P^{2} / m_{0}=18.71 \mathrm{eV},{ }^{22}$ we have calculated the squared optical transition-matrix elements from the first electronic energy level (CB1) (where CB denotes conduction band) to the first LH energy level (LH1) and the first HH energy level (HH1). The results are given in Table IV, in comparison with the results of the superlattice model. From our calculations, we found that the optical transitions are clearly anisotropic, and the squared optical
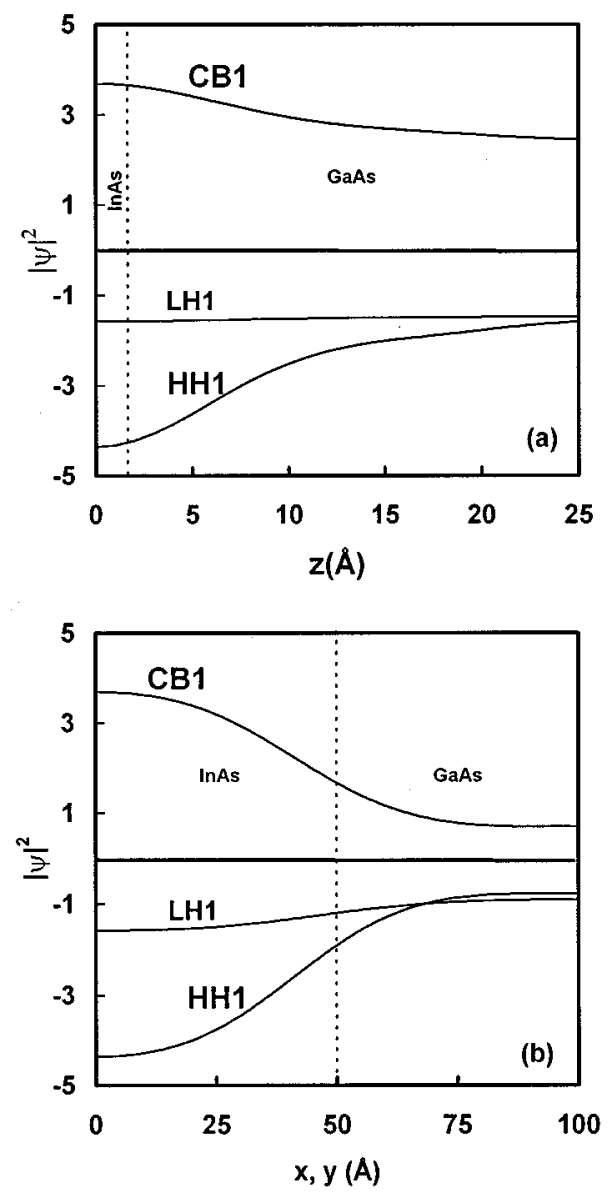

FIG. 3. The distribution of electron and hole wave functions along the coordinate axes. The dotted line is the interface of InAs and GaAs. 
transition-matrix elements of the dot model are close to those of the superlattice mode.

Figure 3 shows the distribution of electron and hole wave functions of the ground states along coordinate axes for coupled InAs monolayer quantum dots for the above structure parameters. Here the upper lines correspond to electrons and the lower lines correspond to holes. From this figure, we see that the wave functions of electrons and holes penetrate very deeply into the GaAs barrier along the growth direction. Also the penetration of the wave functions of $\mathrm{LH}$ into $\mathrm{GaAs}$ is deeper than those of HH. Taking above effective-mass parameters, we can calculate the exciton states. We found that the LH exciton binding energies are very close to the value of bulk GaAs and weakly dependent on the structure parameters of the InAs dots. This is also due to the very deep penetration of the $\mathrm{LH}$ wave functions into the GaAs barrier. For $\mathrm{HH}$, when $d=50 \AA$ and $L=200 \AA$, the binding energies are close to the value of bulk GaAs and weakly dependent on the radius $R$; with a fixed value of $d=50 \AA$ and $R=50 \AA$, and $L$ increasing, the binding energies from the minimum value (close to the value of the bulk GaAs) increases to a maximum (only about $4.5 \mathrm{meV}$ ), and then decreases to a value, again, close to the value of bulk GaAs. The $L$ value corresponding to the maximum binding energy is about 650 $\AA$. When $R$ and $L$ are very large, the results will be similar to the results of InAs/GaAs superlattices. In a word, the excitonic binding energies are close to the value of bulk GaAs.

Figure 4 gives the theoretical absorption spectra from HH1 and LH1 to CB1 for coupled monolayer InAs quantum dots with $d=50 \AA, R=50 \AA$, and $L=300 \AA$. These structure parameters are close to the experimental parameters in Ref. 30. For comparison, we note that the photoluminescence experimental data given by Ref. 30 show that HH1 and LH1 exciton absorption peaks are located at 1.502 and $1.5106 \mathrm{eV}$, respectively, and our theoretical absorption peaks are at 1.5025 and $1.5085 \mathrm{eV}$ for the HH1 and LH1 exciton, respectively. Obviously our theoretical results agree well with the experimental data. ${ }^{30}$

*Also at the Department of Physics, Hong Kong University of Science \& Technology, Clear Water Bay, Kowloon, Hong Kong.

${ }^{1}$ M. Sato and Y. Horikoshi, J. Appl. Phys. 66, 851 (1989).

${ }^{2}$ J.-Y. Marzin and J.-M. Gerard, Phys. Rev. Lett. 62, 2172 (1989).

${ }^{3}$ J. H. Lee, K. Y. Hsieh, and R. M. Kolbas, Phys. Rev. B41, 7678 (1990).

${ }^{4}$ E. P. O’Reilly, Semicond. Sci. Technol. 4, 121 (1989).

${ }^{5}$ M. Yazawa, M. Koguchi, and K. Hiruma, Appl. Phys. Lett. 58, 1080 (1991).

${ }^{6}$ O. Brandt, L. Tapfer, K. Ploog, R. Bierwolf, M. Hohenstein, F. Phillip, H. Lage, and A. Heberle, Phys. Rev. B 44, 8043 (1991).

${ }^{7}$ W. J. Scahaffer, M. D. Lind, S. P. Kowalczyk, and R. W. Grant, J. Vac. Sci. Technol. B 1, 688 (1983).

${ }^{8}$ F. Houzay, C. Guille, J. M. Moison, P. Henoc, and F. Barthe, J. Cryst. Growth 81, 67 (1987).

${ }^{9}$ J. F. Carlin, R. Houdre, A. Rudra, and M. Ilegems, Appl. Phys. Lett. 59, 3018 (1991).

${ }^{10}$ C. W. Synder, B. G. Orr, D. Kessler, and L. M. Sander, Phys. Rev. Lett. 66, 3032 (1991).

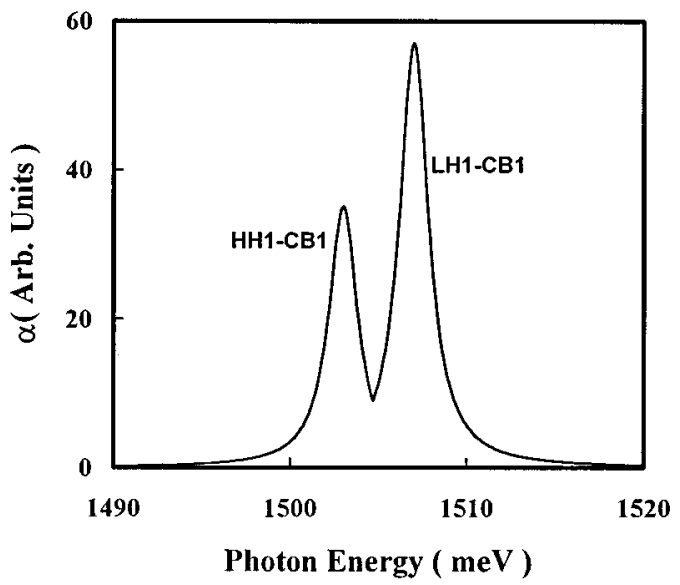

FIG. 4. Theoretical absorption spectra with $l=2.83 \AA, d=50 \AA$, $R=50 \AA$, and $L=300 \AA$.

\section{SUMMARY}

In this paper we have given an effective-mass theory for coupled InAs quantum dots grown on $\mathrm{GaAs}(100)$ oriented substrates. At the $\Gamma$ point, we have calculated the electron and hole energy levels, the distribution of electron and hole wave functions along the growth direction as well as in the plane, the optical transition matrix elements, the excitonic states, the energy transitions as functions of the radius and period of InAs dots, and the optical-absorption spectra. Our theoretical results agree well with the available experimental data.

\section{ACKNOWLEDGMENTS}

This work was supported by the State Key Program for Basic Research and the National Natural Science Foundation of China, and Grant No. MFG 94/95. SC03 at HKUST, and RGC Grant No. 209/93E.
${ }^{11}$ A. Madhukar, Q. Xie, P. Chen, and A. Konkar, Appl. Phys. Lett. 64, 2727 (1994).

${ }^{12}$ D. Leonard, M. Krishnamurthy, S. Fafard, J. L. Merz, and P. M. Petroff, J. Vac. Sci. Technol. B 12, 1063 (1994).

${ }^{13}$ J. M. Moison, F. Houzay, F. Barthe, L. Leprince, E. Andre, and O. Vatel, Appl. Phys. Lett. 64, 196 (1994).

${ }^{14}$ J. M. Marzin, J. M. Gerard, A. Izrael, D. Barrier, and G. Bastard, Phys. Rev. Lett. 73, 716 (1994).

${ }^{15}$ M. Grundmann, J. Christen, N. N. Ledentsov, J. Bohrer, D. Bimberg, S. S. Ruvimov, P. Werner, U. Richter, U. Gosele, J. Heydenreich, V. M. Ustinov, A. Yu. Egorov, A. E. Zhukov, P. S. Kop'ev, and Zh. I. Alferov, Phys. Rev. Lett. 74, 4043 (1995).

${ }^{16}$ S. Fafard, R. Leon, D. Leonard, J. L. Merz, and P. M. Petroff, Phys. Rev. B 50, 8086 (1994).

${ }^{17}$ P. D. Wang, N. N. Ledentsov, C. M. Sotomayer Torres, P. S. Kop'ev, and V. M. Ustinov, Appl. Phys. Lett. 64, 1526 (1994).

${ }^{18}$ Qianghua Xie, Anupan Madhukar, Ping Chen, and Nobuhiko P. Kobayashi, Phys. Rev. Lett. 75, 2542 (1995).

${ }^{19}$ M. G. Burt, J. Phys. Condens. Matter 4, 6651 (1992).

${ }^{20}$ Bradley A. Foreman, Phys. Rev. B 52, 12241 (1995). 
${ }^{21}$ Bradley A. Foreman, Phys. Rev. B 48, 4964 (1993).

${ }^{22}$ Tang Hui and Huang Kun, Chin. J. Semicond. 8, 1 (1987).

${ }^{23}$ Fan Weijun and Xia Jianbai, Chin. J. Semicond. 13, 133 (1992).

${ }^{24}$ K. Suzuki and J. C. Hensel, Phys. Rev. B 9, 4184 (1974).

${ }^{25}$ H. Mathieu, P. Mele, E. L. Ameziane, B. Archila, and J. Camassel, Phys. Rev. B 19, 2209 (1970).

${ }^{26}$ J. M. Luttinger, Phys. Rev. 102, 1030 (1956).

${ }^{27}$ Y. C. Chang and J. N. Schulman, Phys. Rev. B 31, 2069 (1985).
${ }^{28}$ Hanyou Chu and Yia-Chung Chang, Phys. Rev. B 39, 10861 (1989).

${ }^{29}$ Physics of Group IV Elements and III-V Compounds, edited by O. Madelung, Landolt-Bornstein, Numerical Data and Functional Relationships in Science and Technology, New Series, Group III, Volume 17, Pt. a (Springer Verlag, Berlin, 1982).

${ }^{30}$ P. D. Wang, N. N. Ledentsov, C. M. Sotomayer Torres, I. N. Yassievich, A. Pakhomov, A. Yu. Egovov, P. S. Kop'ev, and V. M. Ustinov, Phys. Rev. B 50, 1604 (1994). 